\title{
Origin of the Sharkskin Instability: Nonlinear Dynamics
}

\author{
Stylianos Varchanis $\odot,{ }^{1,2}$ Dionisis Pettas $\odot,{ }^{1}$ Yannis Dimakopoulos $\odot,{ }^{1}$ and John Tsamopoulos $\circledast^{1, *}$ \\ ${ }^{1}$ Laboratory of Fluid Mechanics \& Rheology, Department of Chemical Engineering, University of Patras, Patras 26500, Greece \\ ${ }^{2}$ Micro/Bio/Nanofluidics Unit, Okinawa Institute of Science and Technology, Onna, Okinawa 904-0495, Japan
}

(Received 9 March 2021; revised 25 May 2021; accepted 30 June 2021; published 17 August 2021)

\begin{abstract}
The appearance of surface distortions on polymer melt extrudates, often referred to as sharkskin instability, is a long-standing problem. We report results of a simple physical model, which link the inception of surface defects with intense stretch of polymer chains and subsequent recoil at the region where the melt detaches from the solid wall of the die. The transition from smooth to wavy extrudate is attributed to a Hopf bifurcation, followed by a sequence of period doubling bifurcations, which eventually lead to elastic turbulence under creeping flow. The predicted flow profiles exhibit all the characteristics of the experimentally observed surface defects during polymer melt extrusion.
\end{abstract}

DOI: 10.1103/PhysRevLett.127.088001

The most important process in plastics manufacturing is extrusion, where a polymer melt is forced through a die. It is well known that, as the flow rate increases, the stable flow turns unstable and the smooth extrudate surface becomes increasingly wavy and disordered. The surface in the first observable flow transition consists of semiregular grooves that run mainly perpendicular to the flow direction and resemble the skin of a shark, hence the term sharkskin instability. This has intrigued the scientific and industrial communities for over 60 years. It imposes a limit on the rate of production in polymer processing operations by decreasing the quality of the product. Although it has been studied experimentally in great depth [1-4], the physical mechanisms driving the onset of the instability remain unclear. Three prevailing physical mechanisms have been proposed regarding the onset of the instability: (1) the breakdown of the no-slip condition at the die exit [5-9], (2) a multivalued flow curve (the so-called shear banding), [10-13], and (3) the development of intense tensile stresses at the die exit that force the melt to respond like rubber, causing crack-like scratches on its surface [2,14-18]. There is evidence in favor or against these scenarios. For example, an experimentally observed decrease of slope in shear stress versus shear rate curves at the onset of sharkskin $[5,6]$ favors the slip related theories, because it is interpreted as a loss of friction with the die wall. On the other hand, the addition of slip-promoting agents, which reduce the tensile stresses suppresses the instability $[15,19,20]$. This supports the strong tensile stress theory, opposing the slip theory.

Published by the American Physical Society under the terms of the Creative Commons Attribution 4.0 International license. Further distribution of this work must maintain attribution to the author(s) and the published article's title, journal citation, and DOI.
Simulations with a nonmonotone slip law [9] predicted that the flow becomes unstable with increasing flow rate, and the free surface features periodic oscillations. This favors slip-related theories, but the flow profiles exhibited strong oscillations in the pressure and velocity throughout the die, something that is not related with the sharkskin instability $[2,4,6,21]$. Additionally, there is no experimental evidence to support a nonmonotone slip law in melts which exhibit extrusion instabilities $[3,8,22]$. These are some examples of the numerous studies conducted in an effort to uncover the physical origin of the sharkskin instability. Nevertheless, it is now agreed [2-4] that sharkskin exhibits three main characteristics: (1) It is a surface phenomenon; the flow is distorted and transient only close to the free surface, while away from it the flow is steady and laminar. (2) The periodic distortions have very short wavelength and amplitude compared to the radius or width of the die. (3) The addition of slip-promoting agents on the die wall suppresses the onset of sharkskin.

Regarding earlier theoretical models, the strong stress singularity at the point where the fluid detaches from the wall has posed severe limitations in analyzing this phenomenon. Any analytical attempts $[23,24]$ refer to steady flows. Thus, a numerical solution of the governing equations is necessary. However, this singularity has previously caused loss of convergence near that point and has triggered the well-known high Weissenberg number problem (HWNP) [25]. Moreover, the ratio of length scales between the die cross section and the sharkskin distortions is over 2 orders of magnitude, requiring an extraordinary computational cost and time for the accurate solution of the model. Consequently, theoretical works so far have been performed in unrealistic flow conditions $[9,17,18,26]$ and have never captured the sharkskin instability.

In this Letter, we present a simple model that captures the essential physics during polymer extrusion. We model the 


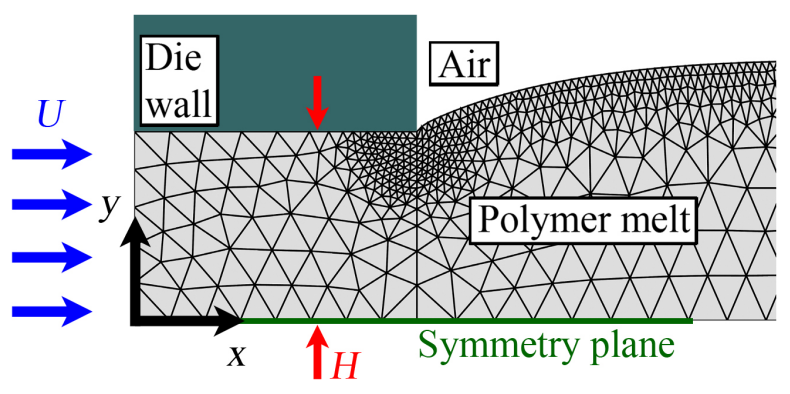

FIG. 1. Schematic of the polymer melt extrusion process and an indicative domain tessellation. For a magnification of the mesh very close to the singularity see Fig. S1.

process as a transient, two-dimensional flow, assuming that the depth of the slit is very long compared to its width (Fig. 1). The melt is incompressible with density $\rho$, interfacial tension $\sigma$, relaxation time $\lambda$, and zero-shear-rate viscosity $\eta_{p}$. The width of the die is denoted as $2 H$. Assuming planar symmetry, only half of the domain is considered. The average velocity of the melt at the entrance of the die is denoted as $U$. At the die exit, the melt suddenly meets the ambient air, forming a three-phase contact line, which is assumed to be pinned, and the contact angle formed by the melt and the solid is determined by the flow field. Cartesian coordinates are employed, with the origin depicted in Fig. 1. At the die wall, we impose the no-slip and no-penetration conditions that change abruptly at the die exit to free surface conditions. At the inflow and outflow boundaries, we impose fully developed flow and an open boundary condition [27], respectively. The die exit is located at $x=1.5 \mathrm{H}$, and the outflow boundary at $x=5 \mathrm{H}$. These lengths do not affect our predictions in any way. We scale all lengths with $H$, all velocities with $U$, and all stresses with the viscous scale $\eta_{p} U / H$. The dimensionless numbers that arise are the Reynolds number $\left(\operatorname{Re}=\rho U H / \eta_{p}\right)$, the Stokes number $\left(\mathrm{St}=\rho g H^{2} / \eta_{p}, g\right.$ : gravitational acceleration), the elastocapillary number $\left(\mathrm{Ec}=\eta_{p} H / \lambda \sigma\right)$, and the Weissenberg number $(\mathrm{Wi}=\lambda U / H)$. In reported experiments $[6,8,15,16,21,28-30], 2 H \sim 0.001 \mathrm{~m}$, and $U \sim 0.01 \mathrm{~m} / \mathrm{s}$, while for a typical melt that produces sharkskin $\eta_{p}>10^{3} \mathrm{Pas}$. Thus, we neglect all inertial $\left(\operatorname{Re}<10^{-5}\right)$ and gravitational $\left(\mathrm{St}<10^{-5}\right)$ forces and directly relate the flow rate with Wi.

The rheology of the melt is described by the exponential Phan-Thien and Tanner (EPTT) model [31], because it can reproduce very well the rheology of entangled polymer melts with the minimum number of parameters [32]. Apart from $\lambda$ and $\eta_{p}$, the parameter $\epsilon$ governs the rheology of the melt: large values $(\epsilon \geq 0.1)$ describe highly entangled melts with intense shear- and extension-thinning effects, while small values $(\epsilon<0.1)$ represent less entangled polymer melts with mild shear-thinning and extension-hardening effects [33] (Fig. S2). For any positive value of $\epsilon$, a strictly monotonic flow curve is predicted, excluding shear banding. Consequently, the process is governed by only three parameters: Wi, Ec, and $\epsilon$.

Recently, we performed a linear stability analysis of this problem [26] and predicted that a Hopf bifurcation occurs at a critical $\mathrm{Wi}$, and the flow transitions from steady to timedependent state [33] (Fig. S4A). We also demonstrated that the source of the disturbance is located at the die exit [33] (Fig. S4B). We did not use slip conditions or a nonmonotone flow curve, but we related the transition to the strain thinning of the melt, due to intense tensile stresses at the die exit. However, numerical issues allowed accessing only low values of $\mathrm{Ec}(\leq 10)$, which do not correspond to melts under processing conditions where Ec $\sim 1000$. Moreover, linear stability analysis gives only an estimation of the shape of distortion (Fig. S4B) without predicting the evolution of the disturbance after the onset of the instability. In the present nonlinear analysis, we perform transient simulations with our stabilized finite-element formulation PEGAFEM$\mathrm{V}[39,40]$, to access the region of the parametric space that corresponds to realistic flow conditions, study the stability of the process, and identify the origin of sharkskin. We emphasize that the new results agree with those of the linear stability analysis [33] (Fig. S4A) and converge with mesh and time step refinement [33] (Tables. S1 and S2).

In what follows we adopt as our base case an EPTT fluid with $\epsilon=0.1$ and $\mathrm{Ec}=1000$. These values of $\epsilon$ and $\mathrm{Ec}$ represent qualitatively [33] (Figs. S2 and S3) the rheology of a broad spectrum of melts under processing conditions $[8,20,21]$. Starting from low values of Wi $(<0.075)$, we find that the flow reaches a steady state, featuring a smooth extrudate surface. For $\mathrm{Wi}>0.075$, the flow reaches a periodic state. Figure 2(a) and Movies S1 and S2 present the flow profile for $\mathrm{Wi}=0.0774$ around the die exit, along with contours of the $x x$ component of the extra stress $\tau_{x x}$. The surface of the extrudate is distorted by traveling waves, starting from the die exit and heading to the outflow. The insets in Fig. 2(a) present the height of the free surface $\left(F_{s}\right)$ very close to the die exit $(x=1.51)$ versus time, and the limit cycle in the phase space defined by $F_{s}, \tau_{x x}$, and $\tau_{x y}$, calculated at $x=1.51$. The solution is periodic, featuring only one frequency. Note that the flow oscillates only close to the free surface, while it is steady away from it. The contours of $\tau_{x x}$ reveal very high stress values in a limited region around the singularity, indicating significant chain stretch.

Having identified the transition from steady to periodic flow, due to a supercritical Hopf bifurcation, in agreement with linear stability [26], we proceed to higher values of Wi. Up to $\mathrm{Wi}=0.0775$, the flow reaches periodic states with a single frequency; shortly after that value of Wi, the system reaches a periodic state that features two discrete frequencies [Fig. 2(b), Movies S3 and S4]. The free surface is more distorted, featuring disturbances with greater amplitude, and the period of the oscillation has been almost 


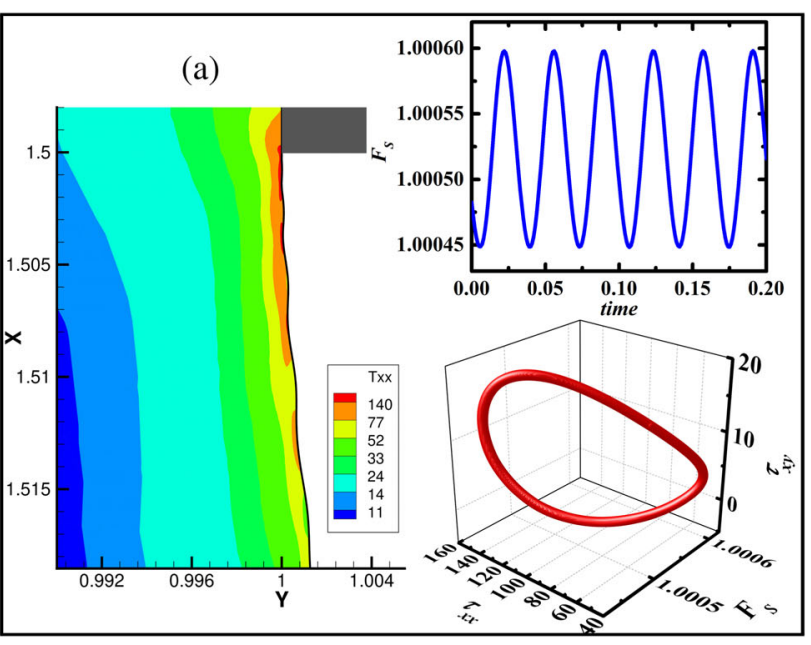

Hopf bifurcation: $W i=0.075$ (b)

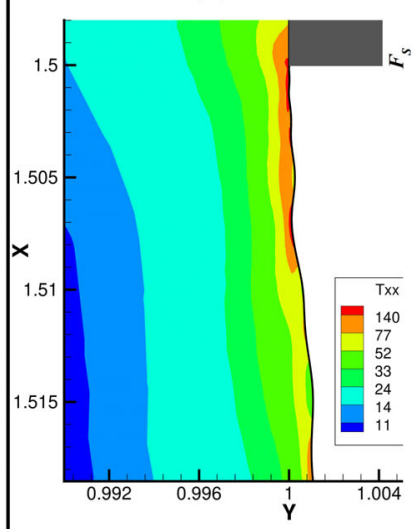

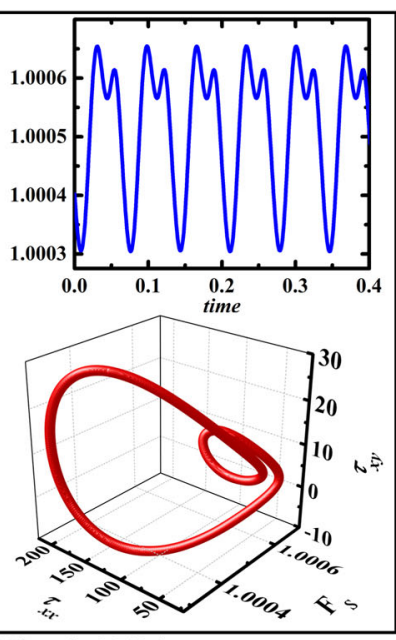

$2^{\text {nd }}$ period doubling: $W i=0.0781$
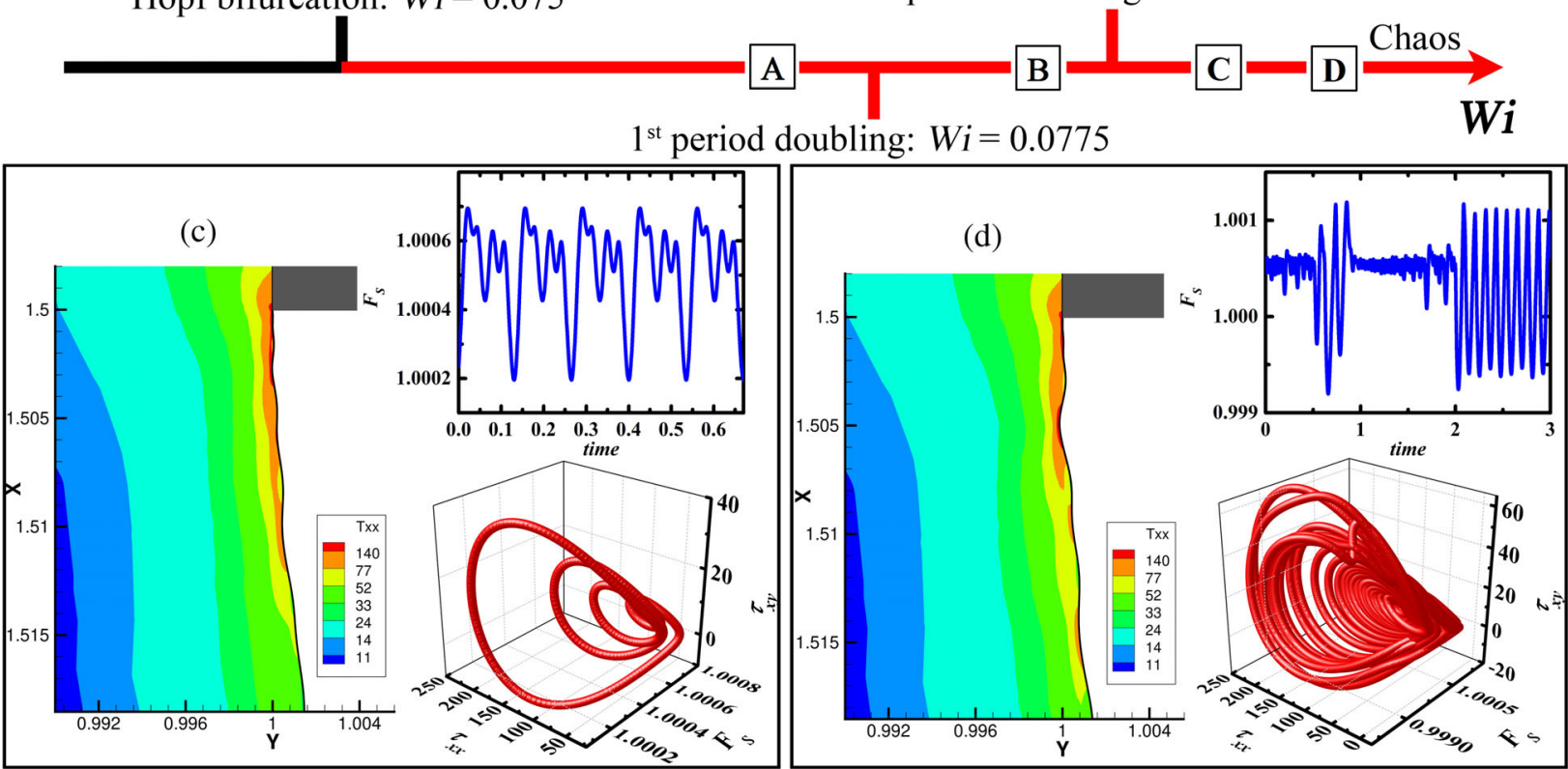

FIG. 2. The route to elastic turbulence for $\epsilon=0.1$ and $\mathrm{Ec}=1000$. Flow profiles for various Wi close to the die exit, along with the free surface height $\left(F_{s}\right)$ close to the die exit $(x=1.51)$ versus time, and the limit cycle in the phase space defined by $F_{s}, \tau_{x x}$, and $\tau_{x y}$, calculated at $x=1.51$. (a) Wi $=0.0774$ (single frequency), (b) Wi $=0.07808$ (two frequencies), (c) $\mathrm{Wi}=0.07829$ (four frequencies), (d) $\mathrm{Wi}=0.07836$ (chaotic). For the spatial form of the wave see [33].

doubled. Clearly, a period-doubling bifurcation has occurred. An additional very small increase in Wi leads to a new flow configuration [Fig. 2(c), Movies S5 and S6]. At $\mathrm{Wi}=0.07829$, four discrete frequencies appear. A period-doubling bifurcation has taken place again, as depicted in the shape of the limit cycle. The free surface is very wavy, but still periodic. Increasing Wi further, we arrive at a flow configuration with no discrete frequencies for $\mathrm{Wi}=0.07836$ [Fig. 2(d), Movies S7 and S8]. The signal is chaotic, while the limit cycle has turned into a strange attractor. In other words, the flow is now turbulent. To be more precise, we find that elastic turbulence under creeping flow $(\operatorname{Re}=0)$ is localized close to the free surface.

This phenomenon is a usual route to chaos via a sequence of period-doubling bifurcations. It is noteworthy that the predicted ratio $\left(\mathrm{Wi}_{c 3}-\mathrm{Wi}_{c 2}\right) /\left(\mathrm{Wi}_{c 2}-\mathrm{Wi}_{c 1}\right)=$ 4.1667 approximates the first Feigenbaum constant $(\delta=4.6692 \ldots)$, as it should during a route to chaos. Increasing surface complexity of the extrudate has been observed experimentally [41-44], but has never been attributed to a sequence of period-doubling bifurcations that lead to elastic turbulence. Moreover, our predictions regarding length scales and $\mathrm{Wi}_{c 1}<0.1$ agree with experiments for monodisperse melts $[20,21]$. The spatial form of the wave, along with qualitative comparisons to experimental data are given in [33]. Finally, to ensure that the predicted instability is the sharkskin, we verified that adding slip to the die wall suppresses the instability [33].

Having answered the question: How does the smooth extrudate become wavy? we proceed to a quest for an 

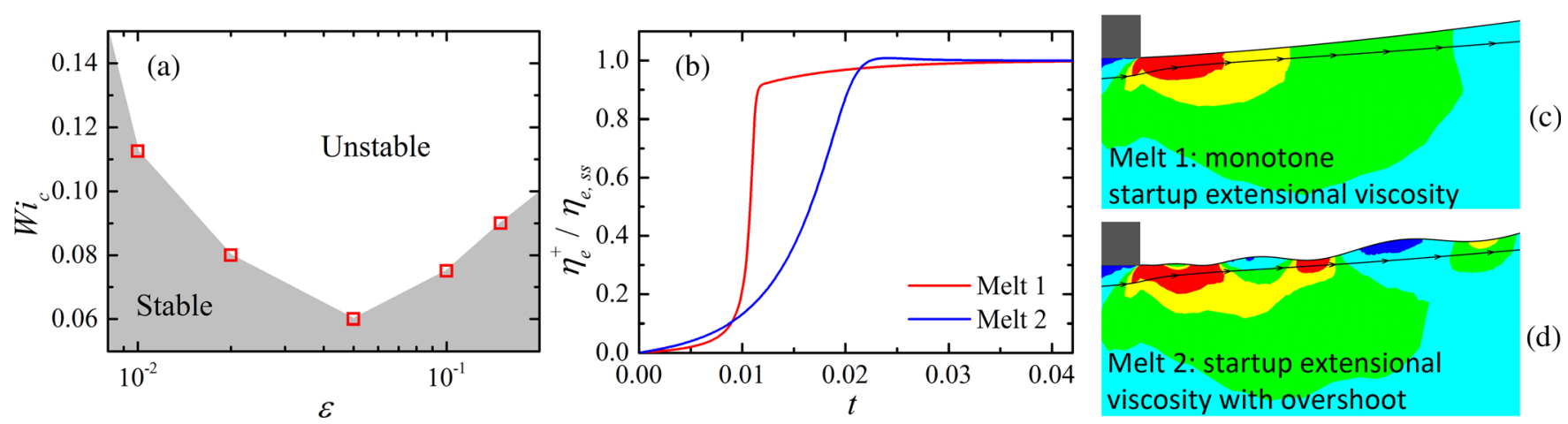

FIG. 3. (a) Critical Weissenberg number $\left(\mathrm{Wi}_{c}\right)$ versus $\epsilon$. (b) Startup extensional viscosities versus time $(t)$ of polymer melts 1 and 2 at extension rate $100 \mathrm{~s}^{-1}$, normalized by the steady value of the extensional viscosity at the same extension rate. (c) and (d) Flow profiles near the die exit for polymer melts 1 and 2, with a streamline superimposed on contours of the $x x$ component of the deformation rate tensor.

answer to the question: Why does this happen? Thus, we investigate the effect of the melt rheology on the stability of the process. Figure 3(a) presents the stability map in the parameter space defined by $\mathrm{Wi}$ and $\epsilon$. Low values of $\epsilon$ stabilize the flow, while intermediate values destabilize it. The minimum is observed because as $\epsilon$ is increased beyond it, the fluid becomes increasingly strain thinning, with capillarity gaining ground and stabilizing the flow. As $\epsilon \rightarrow 0$, the fluid ceases to exhibit shear thinning and becomes infinitely extension hardening (EPTT model reduces to the upper-convected Maxwell (UCM) model when $\epsilon=0$ ). In the limit of the UCM model the flow reaches a steady state with no surface distortions. These observations are consistent with experiments [33,45,46], which reported that polymers exhibiting long chain branching and strain-hardening effects $(\epsilon \rightarrow 0)$ are less sensitive to sharkskin. Moreover, the linear stability analysis of the extrusion process with dominant and finite capillary effects $[18,26]$, revealed that strain thinning has a destabilizing effect. However, strain thinning refers both to shear and extension thinning, but each effect has a very different impact on the flow. To distinguish between them, we performed simulations with the linear Phan-Thien and Tanner (LPTT) model, which predicts shear thinning, but extension hardening [33] (Fig. S7). In all cases with the LPTT model we find a steady state, without surface oscillations [33] (Fig. S8A). Consequently, extension thinning plays a crucial role in the onset of sharkskin. Although this is a notable conclusion, it does not constitute a physical mechanism for flow instability. Moreover, it does not resolve all issues because it suggests that any melt with extension thinning will present sharkskin.

To test whether any melt exhibiting extension thinning can produce sharkskin, we studied another constitutive model (finite extensible Giesekus, FEG [47]) that yields similar predictions with the EPTT model in steady shear and uniaxial extension [33] (Fig. S7). To our surprise, the flow always resulted in a steady state with smooth extrudate surface [33] (Fig. S8B). This turned our attention to dynamic rheological properties. In transient elongation, we observe that the EPTT model predicts an overshoot in the startup extensional viscosity just before steady state is reached under high extension rates [Fig. 3(b)]. This overshoot can be interpreted as a chain recoil after elongation. This response is observed in experiments of polymer melts, but is an unusual feature of a constitutive model, as their vast majority predicts a monotone startup extensional viscosity. Keeping this in mind, we propose the following physical mechanism: (1) If the melt exhibits extension thinning, reduced flow resistance arises around the die exit. (2) With the melt becoming rheologically softer and more prone to deformation, this chain recoil effect after the abrupt elongation at the die exit decelerates the flow, causing nonmonotonicity in the velocity gradient and, thus the distortions are formed.

This physical mechanism is depicted in Figs. 3(b)-3(d) and Movie S9. Figure 3(b) presents the startup extensional viscosity of melt 1 (FEG, red curve), which does not exhibit an overshoot, and melt 2 (EPTT model, blue curve) which does. Following the path of a fluid parcel that flows near the singularity, the displacement along a streamline [Figs. 3(c) and $3(\mathrm{~d})$ ] corresponds to time in the homogenous extension experiment [(Fig. 3(b)]. In melt 1, the chains relax monotonically after being exposed to the extreme extensional flow field at the die exit. On the contrary, the chains of melt 2 recoil after passing the die exit, interfering with the flow, causing deceleration in the $x$ direction, which in turn causes acceleration in the $y$ direction (mass conservation), and the waves are formed. The melt that flows away from the singularity is not exposed to high extension rates, and therefore, it does not present significant extension thinning and recoil during elongation, relaxing monotonically as it exits the die. This mechanism also explains why the distortions take place only close to the surface of the extrudate.

We demonstrated that the root of the sharskin instability lies in the variation of polymer chain conformations under extensional flow. We propose that the combination of extension thinning of the polymer melt in its bulk and 
recoil of the polymer chains in transient elongation is the only prerequisite for the onset of surface distortions. According to the results of our simple physical model, which is based on principles and methods of continuum mechanics, we believe that we have captured the essence and explained the mystery behind the long-disputed sharkskin instability. The present work advances the fundamental understanding of the relation between the microscopic conformations of polymer chains with macroscopically observed phenomena and opens the way for studying distortions that arise at even higher flow rates, such as the stick-slip instability and the gross melt fracture.

The authors acknowledge stimulating discussions with M. M. Denn, G. H. McKinley, and G. Karapetsas. The authors also acknowledge T. Akylas and S. J. Haward for discussing the manuscript. Financial support has been provided by HFRI, Grant No. FM17-2309.

*tsamo@chemeng.upatras.gr

[1] C. J. Petrie and M. M. Denn, Instabilities in polymer processing, AIChE J. 22, 209 (1976).

[2] M. M. Denn, Extrusion instabilities and wall slip, Annu. Rev. Fluid Mech. 33, 265 (2001).

[3] S. G. Hatzikiriakos and K. B. Migler, Polymer Processing Instabilities: Control and Understanding (CRC Press, New York, 2004).

[4] R. Koopmans, J. Den Doelder, and J. Molenaar, Polymer Melt Fracture (CRC Press, Boca Raton, 2010).

[5] A. Ramamurthy, Wall slip in viscous fluids and influence of materials of construction, J. Rheol. 30, 337 (1986).

[6] D. S. Kalika and M. M. Denn, Wall slip and extrudate distortion in linear low-density polyethylene, J. Rheol. 31, 815 (1987).

[7] D. A. Hill, T. Hasegawa, and M. M. Denn, On the apparent relation between adhesive failure and melt fracture, J. Rheol. 34, 891 (1990).

[8] S. G. Hatzikiriakos and J. M. Dealy, Wall slip of molten high density polyethylenes. II. Capillary rheometer studies, J. Rheol. 36, 703 (1992).

[9] E. Brasseur, M. M. Fyrillas, G. C. Georgiou, and M. J. Crochet, The time-dependent extrudate-swell problem of an oldroyd-b fluid with slip along the wall, J. Rheol. 42, 549 (1998).

[10] M. M. Denn, Issues in viscoelastic fluid mechanics, Annu. Rev. Fluid Mech. 22, 13 (1990).

[11] R. G. Larson, Instabilities in viscoelastic flows, Rheol. Acta 31, 213 (1992).

[12] J. D. Shore, D. Ronis, L. Piché, and M. Grant, Theory of melt fracture instabilities in the capillary flow of polymer melts, Phys. Rev. E 55, 2976 (1997).

[13] A. L. Yarin and M. D. Graham, A model for slip at polymer/ solid interfaces, J. Rheol. 42, 1491 (1998).

[14] F. Cogswell, Stretching flow instabilities at the exits of extrusion dies, J. Non-Newtonian Fluid Mech. 2, 37 (1977).

[15] N. El Kissi, J.-M. Piau, and F. Toussaint, Sharkskin and cracking of polymer melt extrudates, J. Non-Newtonian Fluid Mech. 68, 271 (1997).
[16] K. B. Migler, Y. Son, F. Qiao, and K. Flynn, Extensional deformation, cohesive failure, and boundary conditions during sharkskin melt fracture, J. Rheol. 46, 383 (2002).

[17] R. Keunings, Advances in the computer modeling of the flow of polymetric liquids, Comput. Fluid Dyn. J. 9, 449 (2000).

[18] G. Karapetsas and J. Tsamopoulos, On the stick-slip flow from slit and cylindrical dies of a Phan-Thien and tanner fluid model. ii. linear stability analysis, Phys. Fluids 25, 093105 (2013).

[19] V. G. Ghanta, B. L. Riise, and M. M. Denn, Disappearance of extrusion instabilities in brass capillary dies, J. Rheol. 43, 435 (1999).

[20] G. M. Wise, M. M. Denn, A. T. Bell, J. W. Mays, K. Hong, and H. Iatrou, Surface mobility and slip of polybutadiene melts in shear flow, J. Rheol. 44, 549 (2000).

[21] F. J. Lim and W. Schowalter, Wall slip of narrow molecular weight distribution polybutadienes, J. Rheol. 33, 1359 (1989).

[22] S. Hatzikiriakos and J. Dealy, Wall slip of molten high density polyethylene. I. Sliding plate rheometer studies, J. Rheol. 35, 497 (1991).

[23] R. Tanner, A theory of die-swell, J. Polym. Sci., A-2, Polym. Phys. 8, 2067 (1970).

[24] J. Vlachopoulos, M. Horie, and S. Lidorikis, An evaluation of expressions predicting die swell, Trans. Soc. Rheol. 16, 669 (1972).

[25] M. Alves, P. Oliveira, and F. Pinho, Numerical methods for viscoelastic fluid flows, Annu. Rev. Fluid Mech. 53, 509 (2021).

[26] D. Pettas, G. Karapetsas, Y. Dimakopoulos, and J. Tsamopoulos, On the origin of extrusion instabilities: Linear stability analysis of the viscoelastic die swell, J. NonNewtonian Fluid Mech. 224, 61 (2015).

[27] T. C. Papanastasiou, N. Malamataris, and K. Ellwood, A new outflow boundary condition, Int. J. Numer. Methods Fluids 14, 587 (1992).

[28] N. El Kissi and J. Piau, Adhesion of linear low density polyethylene for flow regimes with sharkskin, J. Rheol. 38, 1447 (1994).

[29] D. Arda and M. Mackley, The effect of die exit curvature, die surface roughness and a fluoropolymer additive on sharkskin extrusion instabilities in polyethylene processing, J. Non-Newtonian Fluid Mech. 126, 47 (2005).

[30] T. I. Burghelea, H. J. Griess, and H. Münstedt, Comparative investigations of surface instabilities (sharkskin) of a linear and a long-chain branched polyethylene, J. Non-Newtonian Fluid Mech. 165, 1093 (2010).

[31] N. P. Thien and R. I. Tanner, A new constitutive equation derived from network theory, J. Non-Newtonian Fluid Mech. 2, 353 (1977).

[32] S. Varchanis, Y. Dimakopoulos, and J. Tsamopoulos, Evaluation of tube models for linear entangled polymers in simple and complex flows, J. Rheol. 62, 25 (2018).

[33] See Supplemental Material at http://link.aps.org/ supplemental/10.1103/PhysRevLett.127.088001 for information about the governing equations, the boundary conditions, the constitutive models, and the numerical method, which includes Refs. [34-37]. 
[34] J. Schöberl, Netgen an advancing front $2 \mathrm{~d} / 3 \mathrm{~d}-$-mesh generator based on abstract rules, Comput. Visual. Sci. 1, 41 (1997).

[35] A. Bach, H. K. Rasmussen, and O. Hassager, Extensional viscosity for polymer melts measured in the filament stretching rheometer, J. Rheol. 47, 429 (2003).

[36] J.-M. Piau, N. El Kissi, F. Toussaint, and A. Mezghani, Distortions of polymer melt extrudates and their elimination using slippery surfaces, Rheol. Acta 34, 40 (1995).

[37] H. K. Rasmussen, J. K. Nielsen, A. Bach, and O. Hassager, Viscosity overshoot in the start-up of uniaxial elongation of low density polyethylene melts, J. Rheol. 49, 369 (2005).

[38] Q. Huang, M. Mangnus, N. J. Alvarez, R. Koopmans, and O. Hassager, A new look at extensional rheology of lowdensity polyethylene, Rheol. Acta 55, 343 (2016).

[39] S. Varchanis, A. Syrakos, Y. Dimakopoulos, and J. Tsamopoulos, A new finite element formulation for viscoelastic flows: Circumventing simultaneously the lbb condition and the high-Weissenberg number problem, J. Non-Newtonian Fluid Mech. 267, 78 (2019).

[40] S. Varchanis, A. Syrakos, Y. Dimakopoulos, and J. Tsamopoulos, Pegafem-v: A new petrov-galerkin finite element method for free surface viscoelastic flows, J. Non-Newtonian Fluid Mech. 284, 104365 (2020).

[41] S.-Q. Wang, P. A. Drda, and Y.-W. Inn, Exploring molecular origins of sharkskin, partial slip, and slope change in flow curves of linear low density polyethylene, J. Rheol. 40, 875 (1996).

[42] E. Miller and J.P. Rothstein, Control of the sharkskin instability in the extrusion of polymer melts using induced temperature gradients, Rheol. Acta 44, 160 (2004).

[43] H.-y. Lee, D. H. Kim, and Y. Son, Anomalous rheological behavior of polyethylene melts in the gross melt fracture regime in the capillary extrusion, Polymer 47, 3929 (2006).

[44] C. Tzoganakis, B. C. Price, and S. G. Hatzikiriakos, Fractal analysis of the sharkskin phenomenon in polymer melt extrusion, J. Rheol. 37, 355 (1993).

[45] C. Venet and B. Vergnes, Experimental characterization of sharkskin in polyethylenes, J. Rheol. 41, 873 (1997).

[46] P. J. Doerpinghaus and D. G. Baird, Comparison of the melt fracture behavior of metallocene and conventional polyethylenes, Rheol. Acta 42, 544 (2003).

[47] A. N. Beris and B. J. Edwards, Thermodynamics of Flowing Systems: With Internal Microstructure (Oxford University Press on Demand, New York, 1994). 